\title{
False positive seroreactivity to brucellosis in tuberculosis patients: a prevalence study
}

This article was published in the following Dove Press journal:

International Journal of General Medicine

I I March 201 I

Number of times this article has been viewed

\author{
Mojtaba Varshochi ${ }^{1,2}$ \\ Jafar Majidi² \\ Marjan Amini' \\ Kamyar Ghabili ${ }^{3}$ \\ Mohammadali M Shoja ${ }^{3}$ \\ 'Department of Infectious Disease, \\ Tabriz University of Medical \\ Sciences, Tabriz, Iran; ${ }^{2}$ Infectious \\ Disease and Tropical Medicine \\ Research Center, Tabriz University \\ of Medical Sciences, Tabriz, Iran; \\ ${ }^{3}$ Tuberculosis and Lung Disease \\ Research Center, Tabriz University \\ of Medical Sciences, Tabriz, Iran
}

Background: The rising worldwide incidence of tuberculosis (TB) increases the demand for knowledge about its potential seroreactivity with other microbial agents. A few reports and the authors' experiences indicate that tuberculosis may result in a false-positive brucellosis serology. This may cause a diagnostic challenge because of the close clinical resemblance of these two infections.

Objective: The aim of the present prevalence study was to elucidate brucellosis seroreactivity in patients with active TB.

Methods: Ninety-eight patients with newly diagnosed and active TB were studied using an enzyme-linked immunosorbent assay (ELISA) and Wright's and Coombs-Wright's tests. Seventy-five healthy individuals were used as controls. The patients showed signs of recovery after starting a standard anti-TB regimen and had no clinical evidence of brucellosis at a subsequent 6-month follow-up. The data were analyzed statistically by Fisher's exact test using SPSS 11.0.

Results: We found that $9.2 \%$ of TB patients versus $1.3 \%$ of healthy controls had positive results on the anti-Brucella IgG ELISA $(P=0.04)$. Five TB patients were found to have agglutination on Wright's tests, while none of the controls showed agglutination.

Conclusion: Active TB patients may have some seroreactivity with Brucella antigens, and Brucella IgG ELISA may give a false positive in these patients. Clinicians should consider false positive brucellosis seroreactivity in patients with active TB.

Keywords: false positive serology, ELISA, diagnosis

\section{Introduction}

Brucellosis and tuberculosis (TB) are two important public health hazards of particular concern in developing countries and the Middle East. ${ }^{1}$ These two infectious diseases have some overlapping clinical features; hence, diagnosis relies mainly on paraclinical studies. Brucellosis serological tests are reported to have high sensitivity. ${ }^{2,3}$ However, their specificity is limited by antigenic cross-reactivity and the false positive results encountered in infections with Salmonella, Francisella, Vibrio cholerae, Yersinia enterocolitica, Serratia marcescens, Haemophilus influenzae, Pseudomonas aeruginosa, group A beta-hemolytic Streptococci and Escherichia coli, and the malaria parasite..$^{1,2,4-9}$ The specificity of these serological tests is particularly important during a program of Brucella eradication. ${ }^{4}$

The Centers for Disease Control and Prevention has reported a false-positive Brucella antibody assay in a patient with constitutional symptoms mimicking brucellosis. Because the public health consequences of such a false-positive test
Correspondence: Mohammadali M Shoja Tuberculosis and Lung Disease Research Center, Tabriz University of Medical Sciences, Tabriz, Iran Tel/Fax +98 4II 4438523 Email shoja.m@gmail.com 
can be serious, clinicians and public health professionals should have a thorough knowledge of Brucella serological cross-reactions. Anecdotally, we have had several patients with features of spinal osteomyelitis, vertebral collapse and fever of unknown origin, in whom the initial serological studies showed a considerable titer of anti-Brucella antibodies. However, those patients showed no clinical improvement with Brucella chemotherapy, and surprisingly, later investigations yielded a diagnosis of TB. The literature describes other cases of the involvement of brucellosis, TB, or both in spinal infection, ${ }^{1,10}$ and in other conditions such as meningitis ${ }^{11}$ and primary peritonitis, ${ }^{12}$ as well as fevers of unknown origin. ${ }^{13}$

With the increasing worldwide incidence of TB, in the era of human immunodeficiency (HIV) infection, knowledge about potential seroreactivity associated with TB may be of paramount diagnostic value. The aim of this study was to evaluate false-positive brucellosis seroreactivity detected by enzyme-linked immunosorbent assay (ELISA), Wright's or standard tube agglutination (STA) tests, and Coombs-Wright's tests in patients with active TB.

\section{Methods}

\section{Patient selection}

The prevalence study included 98 consecutive patients with newly diagnosed TB referred to a university-affiliated center, the Tuberculosis and Lung Disease Research Center, between April 2003 and July 2004. Informed consent was obtained from the patients ahead of the study. All the patients had a bacteriological (acid-fast bacilli [AFB] smear plus culture) or histopathological (for some forms of extrapulmonary TB) diagnosis ofTB. Exclusion criteria were age less than 12 years, past history of brucellosis or a known malignancy, intravenous drug abuse, HIV infection, and exposure to domestic animals or their products. Age, gender, clinical category of TB (pulmonary versus extrapulmonary; Table 1), the clinically symptomatic period, and purified protein derivative test results were recorded for each patient. An age- and sex-matched control group of 75 healthy individuals without history of brucellosis, tuberculosis, or any systemic disease was recruited during the same period from potential kidney donors referred to the central university hospital for examination. The institutional board review and ethics committee approved the protocol.

\section{Blood collection and analysis}

A $5 \mathrm{~mL}$ venous blood sample was drawn from each patient. Serum was separated by centrifugation at $750 \times g$ for 10 minutes at room temperature and was then stored at $-20^{\circ} \mathrm{C}$
Table I Clinical features of TB patients with reference to their brucellosis serorectivity

\begin{tabular}{lll}
\hline & All patients $(\mathbf{n})$ & $\begin{array}{l}\text { Patients with false } \\
\text { positive ELISA (n) }\end{array}$ \\
\hline Pulmonary TB & 83 & 7 \\
Cervical adenitis & 8 & 1 \\
Renal TB & 2 & 0 \\
Lupus vulgaris & $\mathrm{I}$ & 0 \\
Genitourinary TB & 3 & 0 \\
Intestinal TB & $\mathrm{I}$ & 0 \\
Pleuritis & $\mathrm{I}$ & $\mathrm{I}$ \\
Peritonitis & $\mathrm{I}$ & 0 \\
\hline
\end{tabular}

Note: Two patients had concomitant TB of two organs.

Abbreviations: ELISA, enzyme-linked immunosorbent assay; TB, tuberculosis.

pending analysis. All the serum samples were analyzed for anti-Brucella antibodies by ELISA and Wright's test. A Coombs-Wright's test was also performed if agglutination was seen on Wright's test, as well as randomly in 18 patients who had negative Wright's tests.

A Brucella IgG ELISA kit (IBL, Hamburg, Germany) was used to titrate the serum anti-Brucella IgG as described by Cox. ${ }^{14}$ Briefly, $1 \mu \mathrm{L}$ of serum was diluted $1: 101$, and after buffer-washing, $100 \mu \mathrm{L}$ of horseradish peroxidase-conjugated anti-human IgG was added. The mixture was incubated for 30 minutes at room temperature and was then treated with tetramethyl benzidine (TMB) for 20 minutes. A Brucella antibody-antigen reaction was indicated by a blue coloration. Subsequently, a TMB stop solution was added, and the optical density of the well was measured with a spectrophotometer (Stat Fax 3200, Awareness Technology, Inc., Palm City, FL, USA); at $450 \mathrm{~nm}$. A serum titer of more than $12 \mathrm{U} / \mathrm{mL}$ was considered positive.

The Wright's test was performed according to Edwards et al. ${ }^{15}$ Briefly, $1 \mathrm{~mL}$ doubling dilutions of serum were made, beginning with a dilution of 1:40. A $0.05 \mathrm{~mL}$ aliquot of concentrated Brucella antigen (Pasteur Institute, Tehran, Iran) was added to each diluted sample. The titer of the serum was recorded as the last tube showing agglutination readily visible to the naked eye after gentle shaking. For the Coombs-Wright's test, the tubes that were negative on the Wright's test were centrifuged, and the pellets were washed. After adding a drop of anti-human globulin (Coombs reagent; Cinagen, Tehran, Iran), the tubes were incubated at $37^{\circ} \mathrm{C}$ for 30 minutes. Agglutination was assessed after a final centrifugation and was quantified as the last positive tube.

\section{Data analysis}

All data were presented as mean \pm standard deviation. The data were analyzed statistically by Fisher's exact test using 
SPSS software (v. 11.0; SPSS Inc, Chicago, IL). A $P$ value less than 0.05 was considered statistically significant.

\section{Results}

The mean age of the patients was $50.8 \pm 19.7$ years (range 14-84). There were 45 (46\%) female and 53 (54\%) male patients. Eighty-five percent of the patients had pulmonary TB and 15\% had extrapulmonary TB. The mean period during which the patients had been symptomatic was 217.5 days. Table 1 details the clinical features of the TB patients enrolled in this study.

Nine TB patients $(9.2 \%)$ and one healthy control $(1.3 \%)$ gave positive results on the anti-Brucella IgG ELISA (two-tailed $P=0.044$ ). Five of the TB patients, but none of the healthy controls, showed agglutination on Wright's test. The Wright's agglutinin titer ranged between 1:40 and 1:80. The Coombs-Wright's test titers ranged between 1:80 and 1:160 for those patients who had shown agglutination on Wright's test. None of the 18 random samples showed agglutination on the Coombs-Wright's test.

\section{Discussion}

The present study revealed that the anti-Brucella IgG ELISA gave a significant false-positive rate in active TB patients. The Brucella ELISA test is generally believed to have a higher sensitivity and specificity in determining Brucella-specific antibodies than other serological tests., ${ }^{2,16-21}$ Memish et al compared the standard tube agglutination test and ELISA results in brucellosis patients, and in contrast to the above-referenced studies, reported that the sensitivity and specificity of ELISA IgG (45.6\% and 97.1\%, respectively) were lower than those of the standard tube agglutination test $\left(95.6 \%\right.$ and $100.0 \%$, respectively). ${ }^{22}$ Cakan et al found that the ELISA test for brucellosis was more sensitive when both IgG and IgM were used, but the titer value alone did not represent disease status. ${ }^{23}$

In the present study, 5 out of 98 TB patients showed agglutination on the standard tube agglutination test. Only one patient had a significant positive $(1: 160)$ titer for an endemic area. However, the low titers (1:40-1:80) of the standard tube agglutination test might be important in relation to cross-reactivity. Yildiz et al found crossreactivity of the standard tube agglutination tests with other bacterial infections including Salmonella, Streptococci, and Escherichia coli, but none of their four TB patients showed brucellosis seroreactivity. ${ }^{5}$ The authors concluded that the results of the standard tube agglutination test should be interpreted according to the local endemicity and seropositivity rate of the population. Mert et al also found no false-positive standard tube agglutination results in 20 patients with miliary TB. ${ }^{2}$ In the study of Cetin et al, antibodies for Brucella were reported in $1.8 \%$ of the normal population, $6 \%$ of high-risk individuals, and $6.7 \%$ of patients with Brucella-related complaints. ${ }^{24}$ In the present study, only $1.3 \%$ of the healthy individuals had a positive antibody titer on the Brucella ELISA. Likewise, previous studies have revealed a $\sim 3 \%$ of seroprevalence for brucellosis in the general population of Iran..$^{25,26}$

The diagnosis of brucellosis relies mostly on the results of serological tests in patients with suggestive history and physical findings. Most farming areas in Iran are endemic for brucellosis. Therefore, in clinical practice, Wright's test titers of 1:160 or greater are generally considered positive if the patient is from an endemic region. In the present study, none of the TB patients had a Wright's test titer equal to or greater than 1:160. However, it should be noted that a low titer serology might warrant significance in patients with a spectrum of constitutional symptoms resembling brucellosis and TB. A Coombs-Wright's test titer of 1:160 was encountered in one patient. Although we did not perform a blood or bone marrow culture for brucellosis, a 6-month follow-up showed that all these patients responded dramatically to the World Health Organization (WHO) standard antituberculosis regimen, and none of them developed symptoms or signs suggestive of brucellosis. Moreover, Brucella blood cultures are reportedly positive in $15 \%-35 \%$ of patients with active disease. ${ }^{18}$ We therefore thought that adding this expensive and time-consuming test to the present study would not enhance our results. Polymerase chain reaction (PCR) methods have been developed, and these distinguish clearly between brucellosis and TB infections, but they are also likely to prove expensive and would require thorough training in the relevant technical skills, which may limit their general applicability. ${ }^{27,28}$

Ultimately, active TB patients may show some seroreactivity to Brucella antigens and a Brucella IgG ELISA may be falsely positive in these patients. Clinicians should consider these cross-reactions when interpreting the results of serological tests in TB patients. Future studies with larger numbers of patients and further investigations including culture of the bacteria or PCR are required to clarify the issue further.

\section{Acknowledgments}

This study was supported by a grant from the Tuberculosis and Lung Disease Research Center, Tabriz. The authors are 
grateful to Dr Paul S Agutter for his critical review of this manuscript prior to submission.

\section{Disclosure}

None of the authors has any conflict of interest to declare.

\section{References}

1. Turunc T, Demiroglu YZ, Uncu H, Colakoglu S, Arslan H. A comparative analysis of tuberculous, brucellar and pyogenic spontaneous spondylodiscitis patients. J Infect. 2007;55:158-163.

2. Mert A, Ozaras R, Tabak F, et al. The sensitivity and specificity of Brucella agglutination tests. Diagn Microbiol Infect Dis. 2003;46: 241-243.

3. Araj GF, Lulu AR, Khateeb MI, Saadah MA, Shakir RA. ELISA versus routine tests in the diagnosis of patients with systemic and neurobrucellosis. APMIS. 1988;96:171-176.

4. Mainar-Jaime RC, Munoz PM, de Miguel MJ, et al. Specificity dependence between serological tests for diagnosing bovine brucellosis in Brucella-free farms showing false positive serological reactions due to Yersinia enterocolitica O:9. Can Vet J. 2005;46:913-916.

5. Yildiz F, Tanyel E, Hatipoglu CA, Ertem GT, Tulek N, Oral B. Evaluation of brucella tube agglutination test in patients with brucellosis, patients with bacterial infections other than brucellosis and healthy subjects. Mikrobiyol Bul. 2005;39:211-217.

6. Erdenebaatar J, Bayarsaikhan B, Yondondorj A, et al. Epidemiological and serological survey of brucellosis in Mongolia by ELISA using sarcosine extracts. Microbiol Immunol. 2004;48:571-577.

7. Centers for Disease Control and Prevention (CDC). Public health consequences of a false-positive laboratory test result for BrucellaFlorida, Georgia, and Michigan, 2005. MMWR Morb Mortal Wkly Rep. 2008;57:603-605.

8. Nielsen K, Smith P, Yu WL, Halbert G. Salmonella enterica Serotype Urbana Interference with Brucellosis Serology. J Immunoassay Immunochem. 2007;28:289-296.

9. Thirlwall RE, Commander NJ, Brew SD, Cutler SJ, McGiven JA, Stack JA. Improving the specificity of immunodiagnosis for porcine brucellosis. Vet Res Commun. 2008;32:209-213.

10. Kourbeti IS, Tsiodras S, Boumpas DT. Spinal infections: evolving concepts. Curr Opin Rheumatol. 2008;20:471-479.

11. Karsen H, Karahocagil MK, Irmak H, Demiröz AP. A meningitis case of Brucella and tuberculosis co-infection. Mikrobiyol Bul. 2008;42: 689-694.

12. Demiroğlu YZ, Turunç T, Alişkan H, Colakoğlu S, Arslan H. Primary peritonitis due to brucellosis mimicking tuberculous peritonitis. Turk J Gastroenterol. 2009;20:135-137.
13. Sipahi OR, Senol S, Arsu G, et al. Pooled analysis of 857 published adult fever of unknown origin cases in Turkey between 1990-2006. Med Sci Monit. 2007;13:CR318-CR322.

14. Cox PS. A comparison of the rapid slide and standard tube agglutination tests for brucellosis. Trans R Soc Trop Med Hyg. 1968;62:517-521.

15. Edwards JM, Tannahill AJ, Bradstreet CM. Comparison of the indirect fluorescent antibody test with agglutination, complement-fixation, and Coombs test for Brucella antibody. J Clin Pathol. 1970;23: $161-165$.

16. Coghlan JD, Weir DM. Antibodies in human Brucellosis. Br Med J. 1967;2:269-271.

17. Magee JT. An enzyme-labelled immunosorbent assay for Brucella abortus antibodies. J Med Microbiol. 1980;13:167-172.

18. Gad El-Rab MO, Kambal AM. Evaluation of a Brucella enzyme immunoassay test (ELISA) in comparison with bacteriological culture and agglutination. J Infect. 1998;36:197-201.

19. Hunter SB, Bibb WF, Shih CN, Kaufmann AF, Mitchell JR, McKinney RM. Enzyme-linked immunosorbent assay with major outer membrane proteins of Brucella melitensis to measure immune response to Brucella species. J Clin Microbiol. 1986;24:566-572.

20. Gilbert GL, Hawes LA. The antibody response to Brucella: immunoglobulin response measured by enzyme-linked immunosorbent assay and conventional tests. Aust N Z J Med. 1981;11:40-45.

21. Kiel WK, Khan MY. Analysis of 506 consecutive positive serologic tests for brucellosis in Saudi Arabia. J Clin Microbiol. 1987;25: 1384-1387.

22. Memish ZA, Almuneef M, Mah MW, Qassem LA, Osoba AO. Comparison of the Brucella Standard Agglutination Test with the ELISA IgG and IgM in patients with Brucella bacteremia. Diagn Microbiol Infect Dis. 2002;44:129-132.

23. Cakan G, Bezirci FB, Kacka A, et al. Assessment of diagnostic enzymelinked immunosorbent assay kit and serological markers in human brucellosis. Jpn J Infect Dis. 2008;61:366-370.

24. Cetin ET, Coral B, Bilgiy A. Turkiye de insanda Buruselloz insiansmin saptanmazi. Doga Tr J Medical Sciences. 1990;14:324-334.

25. Marandi AR, Azizi F, Larijani B, Jamshidi HR. Health in Islamic Republic of Iran. Geneva, Switzerland: WHO publication; 1998.

26. Alavi SM, Rafiei A, Nikkhooi A. The effect of lifestyle on brucellosis among nomads in Khuzestan province of Iran. PakJ Med Sci. 2007;23: 358-360.

27. Al Nakkas AF, Wright SG, Mustafa AS, Wilson S. Single-tube, nested PCR for the diagnosis of human brucellosis in Kuwait. Ann Trop Med Parasitol. 2002;96:397-403.

28. Queipo-Ortuño MI, Colmenero JD, Bermudez P, Bravo MJ, Morata P. Rapid differential diagnosis between extrapulmonary tuberculosis and focal complications of brucellosis using a multiplex real-time PCR assay. PLoS One. 2009; 4:e4526.
International Journal of General Medicine

\section{Publish your work in this journal}

The International Journal of General Medicine is an international, peer-reviewed open-access journal that focuses on general and internal medicine, pathogenesis, epidemiology, diagnosis, monitoring and treatment protocols. The journal is characterized by the rapid reporting of reviews, original research and clinical studies across all disease areas.

\section{Dovepress}

A key focus is the elucidation of disease processes and management protocols resulting in improved outcomes for the patient.The manuscript management system is completely online and includes a very quick and fair peer-review system. Visit http://www.dovepress.com/ testimonials.php to read real quotes from published authors. 